\title{
WT1 Analog Peptide Vaccine
}

National Cancer Institute

\section{Source}

National Cancer Institute. WT1 Analog Peptide Vaccine. NCI Thesaurus. Code C64635.

A peptide vaccine comprised of an epitope of human Wilms tumor 1 (WT -1) with potential antineoplastic activity. WT-1, a transcription factor, is overexpressed in most types of leukemia and in some solid cancers. Vaccination with the WT-1 analog peptide vaccine may induce a cytotoxic T-lymphocyte $(C T L)$ response against WT-1 expressing cells, resulting in cell lysis and inhibition of cancer cell proliferation. 